\title{
Measurement of the penetration depth and coherence length of MgB2 in all directions using transmission electron microscopy
}

\author{
Loudon, J. C.; Yazdi, Sadegh; Kasama, Takeshi; Zhigadlo, N. D.; Karpinski, J.
}

Published in:

Physical Review B Condensed Matter

Link to article, DOI:

10.1103/PhysRevB.91.054505

Publication date:

2015

Document Version

Publisher's PDF, also known as Version of record

Link back to DTU Orbit

Citation (APA):

Loudon, J. C., Yazdi, S., Kasama, T., Zhigadlo, N. D., \& Karpinski, J. (2015). Measurement of the penetration depth and coherence length of $\mathrm{MgB}_{2}$ in all directions using transmission electron microscopy. Physical Review $B$ Condensed Matter, 91(5), [054505].4ttps://doi.org/10.1103/PhysRevB.91.054505

\section{General rights}

Copyright and moral rights for the publications made accessible in the public portal are retained by the authors and/or other copyright owners and it is a condition of accessing publications that users recognise and abide by the legal requirements associated with these rights.

- Users may download and print one copy of any publication from the public portal for the purpose of private study or research.

- You may not further distribute the material or use it for any profit-making activity or commercial gain

- You may freely distribute the URL identifying the publication in the public portal 


\title{
Measurement of the penetration depth and coherence length of $\mathrm{MgB}_{2}$ in all directions using transmission electron microscopy
}

\author{
J. C. Loudon, ${ }^{1, *}$ S. Yazdi, ${ }^{2}$ T. Kasama, ${ }^{2}$ N. D. Zhigadlo, ${ }^{3}$ and J. Karpinski ${ }^{3,4}$ \\ ${ }^{1}$ Department of Materials Science and Metallurgy, 27 Charles Babbage Road, Cambridge, CB3 OFS, United Kingdom \\ ${ }^{2}$ Centre for Electron Nanoscopy, Technical University of Denmark, DK-2800 Kongens Lyngby, Denmark \\ ${ }^{3}$ Laboratory for Solid State Physics, ETH Zurich, Otto-Stern-Weg 1, CH-8093, Zurich, Switzerland \\ ${ }^{4}$ Institute of Condensed Matter Physics, EPFL, 1015-Lausanne, Switzerland \\ (Received 16 July 2014; revised manuscript received 30 November 2014; published 5 February 2015)
}

\begin{abstract}
We demonstrate that images of flux vortices in a superconductor taken with a transmission electron microscope can be used to measure the penetration depth and coherence length in all directions at the same temperature and magnetic field. This is particularly useful for $\mathrm{MgB}_{2}$, where these quantities vary with the applied magnetic field and values are difficult to obtain at low field or in the $c$ direction. We obtained images of flux vortices from a $\mathrm{MgB}_{2}$ single crystal cut in the $a c$ plane by focused ion beam milling and tilted to $45^{\circ}$ with respect to the electron beam about the crystallographic $a$ axis. A new method was developed to simulate these images that accounted for vortices with a nonzero core in a thin, anisotropic superconductor and a simplex algorithm was used to make a quantitative comparison between the images and simulations to measure the penetration depths and coherence lengths. This gave penetration depths $\Lambda_{a b}=100 \pm 35 \mathrm{~nm}$ and $\Lambda_{c}=120 \pm 15 \mathrm{~nm}$ at $10.8 \mathrm{~K}$ in a field of $4.8 \mathrm{mT}$. The large error in $\Lambda_{a b}$ is a consequence of tilting the sample about $a$ and had it been tilted about $c$, the errors on $\Lambda_{a b}$ and $\Lambda_{c}$ would be reversed. Thus obtaining the most precise values requires taking images of the flux lattice with the sample tilted in more than one direction. In a previous paper [J. C. Loudon et al., Phys. Rev. B 87, 144515 (2013)], we obtained a more precise value for $\Lambda_{a b}$ using a sample cut in the $a b$ plane. Using this value gives $\Lambda_{a b}=107 \pm 8 \mathrm{~nm}, \Lambda_{c}=120 \pm 15 \mathrm{~nm}, \xi_{a b}=39 \pm 11 \mathrm{~nm}$, and $\xi_{c}=35 \pm 10 \mathrm{~nm}$, which agree well with measurements made using other techniques. The experiment required two days to conduct and does not require large-scale facilities. It was performed on a very small sample, $30 \times 15 \mu \mathrm{m}$ and 200 -nm thick, so this method could prove useful for superconductors where only small single crystals are available, as is the case for some iron-based superconductors.
\end{abstract}

DOI: 10.1103/PhysRevB.91.054505

PACS number(s): 74.25.Uv, 68.37.Lp, 74.25.Ha, 74.70.Ad

\section{INTRODUCTION}

Superconductors have zero electrical resistance and expel magnetic flux from their interiors (the Meissner effect). However, if a sufficiently high magnetic field is applied, flux penetrates by flowing along channels called flux vortices. Each vortex carries one quantum of magnetic flux, $\Phi_{0}=h / 2 e$, where $h$ is Planck's constant and $e$ is the electron charge. They consist of a core with a radius given by the coherence length $\xi$, where the number of carriers (electrons or holes) contributing to superconductivity is suppressed. Electrical supercurrents circulate around the center, diminishing over a radius given by the penetration depth, $\Lambda$. In a conventional superconductor, the coherence length is related to the energy required to excite a carrier out of the superconducting state, $\Delta$, and the velocity of the carriers at the Fermi energy, $v_{F}$, via $\xi=\hbar v_{F} / \pi \Delta$ and the penetration depth is related to the number density of carriers involved in superconductivity, $n_{S}$, and their effective mass, $m^{*}$, via $\Lambda=\sqrt{m^{*} / \mu_{0} n_{S} e^{2}}\left(\mu_{0}\right.$ is the permeability of free space).

In a type-I superconductor, the core exceeds the size over which the supercurrents persist and vortices attract one another as the area of normal (nonsuperconducting) material is minimized if the cores overlap. In a type-II superconductor, the supercurrents persist over a larger radius than the core and the Lorentz force causes vortices to repel one another so they

\footnotetext{
*j.c.loudon@gmail.com
}

form a hexagonal array in an isotropic superconductor. Introducing the Ginzburg-Landau parameter, $\kappa \equiv \Lambda / \xi$ : a type-I superconductor has $\kappa<1 / \sqrt{2}$ and a type-II has $\kappa>1 / \sqrt{2}$.

An anisotropic superconductor has different properties along different crystal axes, $a, b$, and $c$. Most are uniaxial so that $a$ and $b$ are equivalent. The anisotropy in the penetration depth is $\gamma_{\Lambda} \equiv \Lambda_{c} / \Lambda_{a b}$ and in the coherence length it is $\gamma_{\xi} \equiv \xi_{a b} / \xi_{c}$. In a one-band superconductor, where there is one source of carriers contributing to superconductivity, the penetration depth and coherence length are independent of the applied magnetic field and their anisotropies are equal. One method to investigate the penetration depths and coherence lengths in both the $a$ and $c$ directions is to induce flux vortices with their axes normal to the $a c$ plane. The vortex then has an elliptical core surrounded by circulating currents as illustrated in Fig. 1(a).

$\mathrm{MgB}_{2}$ is a rare two-band superconductor [1] discovered in 2001 with a transition temperature $T_{c}=39 \mathrm{~K}$. It is uniaxial with a hexagonal crystal structure [1] (space group 191: $P 6 / \mathrm{mmm}$ ) composed of alternating layers of magnesium and boron with lattice parameters $a=b=3.086 \AA$ and $c=3.542 \AA$. Two bands contribute to superconductivity: the $\sigma$ band associated with bonding from the boron $p_{x y}$ orbitals and the $\pi$ band associated with boron $p_{z}$ orbitals [2]. The $\sigma$ carriers are confined to the $a b$ planes whereas the $\pi$ carriers are delocalized almost isotropically. At low magnetic fields, both $\sigma$ and $\pi$ bands contribute to superconductivity but as the field is increased, the $\pi$ contribution diminishes so that above 
(a)

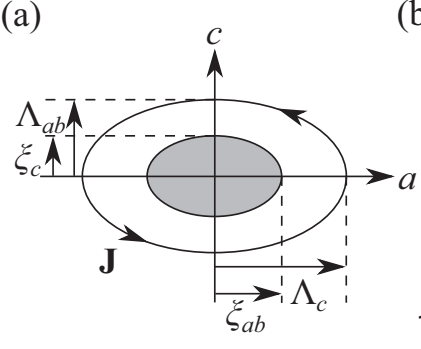

(b)
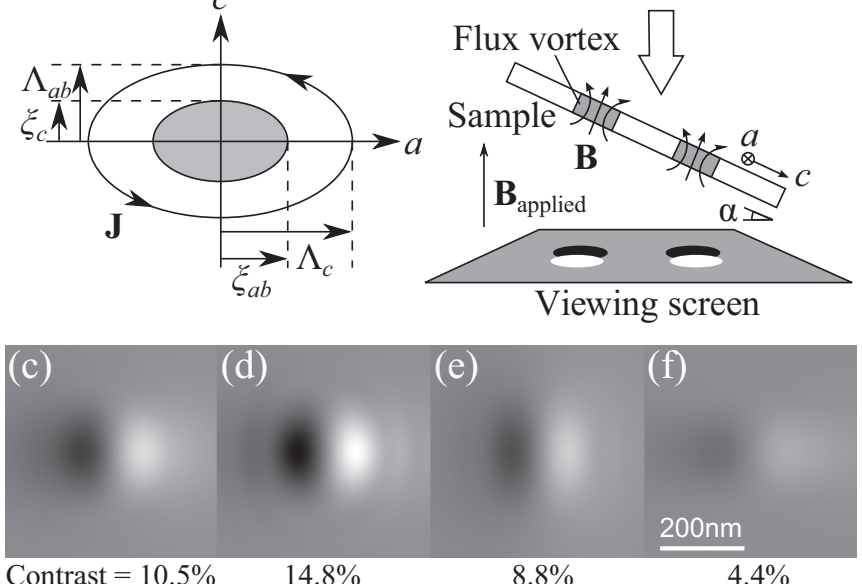

FIG. 1. (a) Flux vortex with its axis normal to the $a c$ plane. The core (shaded) is elliptical with dimensions $\xi_{a b}$ and $\xi_{c}$. Supercurrents $\mathbf{J}$ follow ellipses with the same aspect ratio, diminishing on length scales $\Lambda_{c}$ and $\Lambda_{a b}$. Streamlines of $\mathbf{J}$ are also contours of magnetic flux density B. (b) Experimental arrangement for imaging flux vortices. The electron beam is deflected by the component of $\mathbf{B}$ normal to the beam so vortices appear as black-white features in an out-of-focus image. (c)-(f) Simulated images with defocus $\Delta f=7.15 \mathrm{~mm}$ for a flux vortex in a 180 -nm-thick specimen in the orientation shown in (a) but tilted $45^{\circ}$ about $a$. Contrast values (see text) are shown below each image. (c) $\Lambda_{a b}=100 \mathrm{~nm}, \Lambda_{c}=120 \mathrm{~nm}, \xi_{V}=34 \mathrm{~nm}$, (d) $\Lambda_{a b}=100 \mathrm{~nm}, \Lambda_{c}=120 \mathrm{~nm}, \xi_{V}=1 \mathrm{~nm}$, (e) $\Lambda_{a b}=200 \mathrm{~nm}$, $\Lambda_{c}=120 \mathrm{~nm}, \xi_{V}=34 \mathrm{~nm}$, and (f) $\Lambda_{a b}=100 \mathrm{~nm}, \Lambda_{c}=200 \mathrm{~nm}$, $\xi_{V}=34 \mathrm{~nm}$.

$0.8 \mathrm{~T}$ (at $2 \mathrm{~K}$ ), only the $\sigma$ band contributes [3]. This has the effect that the penetration depth and coherence length vary with field [3].

In a previous paper [4], we showed that the penetration depth of $\mathrm{MgB}_{2}, \Lambda_{a b}$, could be obtained in the low-field limit by making a quantitative comparison between images of flux vortices acquired using transmission electron microscopy and simulations. Here, we extend this method and show that the penetration depths $\Lambda_{a b}$ and $\Lambda_{c}$ and coherence lengths $\xi_{a b}$ and $\xi_{c}$ can be measured in a low field of $4.8 \mathrm{mT}$ from a very small sample.

Focused ion beam milling was used to cut a $\mathrm{MgB}_{2}$ sample in the $a c$ plane of size $30 \times 15 \mu \mathrm{m}$, thinned to $200 \mathrm{~nm}$ so that it was electron transparent (see Sec. III). Flux vortices penetrate normal to the thin surfaces and the sample was tilted about its $a$ axis at $\alpha=45 \pm 5^{\circ}$ to give a component of the $B$ field normal to the electron beam [Fig. 1(b)]. The beam is deflected by the Lorentz force and flux vortices appear as black-white images in an out-of-focus image [5]. Such images are sensitive to the $B$-field throughout the thickness of the specimen, not just the stray field, and they can be acquired in real time [6].

The effect on such images of changing the coherence length and penetration depth is shown in Figs. 1(c)-1(f). These images were simulated by extending Beleggia's method [7] to model vortices with a nonzero core in a thin, anisotropic superconductor (see Sec. II). We use Klemm and Clem's Ginzburg-Landau model for the vortex core [8,9]. In this model, the core has the same symmetry as the circulating currents so that $\xi_{a b} / \xi_{c}=\Lambda_{c} / \Lambda_{a b}$ and $\xi_{V}=\xi_{a b}^{1 / 3} \xi_{c}^{2 / 3}$ but any model for the magnetic structure of a flux vortex could be used with equal facility.

Figure 1(c) shows a simulated image of a vortex with $\Lambda_{a b}=$ $100 \mathrm{~nm}, \Lambda_{c}=120 \mathrm{~nm}$, and $\xi_{V}=34 \mathrm{~nm}$. Panel (d) shows that decreasing $\xi_{V}$ to $1 \mathrm{~nm}$ sharpens the image, increasing the contrast (the difference in the maximum and minimum intensities divided by their sum) from $10.5 \%$ to $14.8 \%$. In (e), $\Lambda_{a b}$ is doubled, which stretches the image in $c$ and reduces the contrast from $10.5 \%$ to $8.8 \%$. Figure 1(f) shows that the images are most sensitive to $\Lambda_{c}$ so that when $\Lambda_{c}$ is increased to $200 \mathrm{~nm}$, the image is stretched in $a$ and its contrast falls to $4.4 \%$. This sensitivity of the images to changes in these parameters should allow the simultaneous measurement of the penetration depths and coherence lengths in all directions. In this paper, we assess the accuracy of this new technique.

\section{SIMULATION OF FLUX VORTEX IMAGES}

In this section, we present a model to calculate the magnetic fields generated by a flux vortex and from this simulate transmission electron micrographs. The model accounts not only for the $B$ field inside the superconductor but also for the spreading of the field lines near the superconductor surface and the field outside. It extends Beleggia's method $[7,10]$ to treat the case of a vortex in a thin, anisotropic superconducting slab and makes use of the work of Klemm and Clem $[8,9,11]$ to account for a nonzero vortex core although it has the convenient feature that any model for the vortex core can be used with equal facility. Like all magnetic objects, flux vortices change only the phase and not the intensity of the electron beam and once the fields have been calculated, the phase shift can be found using the Aharanov-Bohm formula. Once the phase shift is known, any image can be simulated. Thus we first evaluate the magnetic vector potential, then use this to find the phase shift and from this simulate out-of-focus images of flux vortices.

\section{A. Coordinate systems}

In order to visualize flux vortices using transmission electron microscopy, the specimen must be tilted by an angle $\alpha$ to provide a component of the $B$ field normal to the electron beam so that the electrons are deflected by the Lorentz force and show contrast in an out-of-focus image. Thus we follow Beleggia's method $[7,10]$ and introduce two coordinate systems: $X, Y, Z$ referring to the specimen with $X$ and $Y$ in the specimen plane and $Z$ normal to its surface, and $x, y, z$ referring to the microscope with $z$ parallel to the electron beam as illustrated in Fig. 2. The specimen surfaces are at $Z= \pm d$ so its thickness is $t=2 d$. We first evaluate the magnetic vector potential in terms of the specimen coordinates and use this to find the phase shift in the $x y$ plane, which is equivalent to the plane in which images are recorded.

\section{B. Magnetic vector potential}

Here, we evaluate the magnetic vector potential $\mathbf{A}$ from a flux vortex passing through a thin superconducting slab with its axis directed along $Z$, normal to its surfaces. We use 


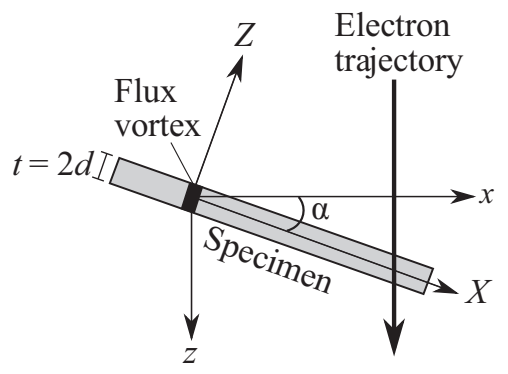

FIG. 2. The relationship between the coordinates $X, Y, Z$ referring to the specimen and the microscope coordinates $x, y, z$. The $y$ and $Y$ axes are normal to the other two axes and point in the direction given by the right-hand rule. The axis of the vortex is parallel to $Z$ and the $B$ field enters the specimen at the bottom and exits at the top. The electron beam is parallel to $z$.

Beleggia's Fourier-space method [10] throughout, in which all the functions are Fourier transformed in $x$ and $y$ but not $z$ (or $X$ and $Y$ but not $Z$ ). This allows the Fourier transform of the vector potential and phase to be expressed by analytical but very lengthy expressions. These were evaluated symbolically using Matlab and only the final inverse transforms were performed numerically. For both coordinate systems, we use the transform convention that if $g(x, y, z)$ is a function in real space, its Fourier transform $\widetilde{g}\left(k_{x}, k_{y}, z\right)$ is

$$
\tilde{g}\left(k_{x}, k_{y}, z\right)=\int_{-\infty}^{\infty} \int_{-\infty}^{\infty} g(x, y, z) e^{-i\left(k_{x} x+k_{y} y\right)} d x d y .
$$

If the order parameter of the superconducting state is written in terms of its amplitude $f$ and phase $\theta$ as $\Psi=f e^{i \theta}$, the vector potential inside the superconductor is related to it by the second Ginzburg-Landau equation [12,13]:

$$
\mathbf{A} f^{2}+L \nabla \times(\nabla \times \mathbf{A})=\frac{\Phi_{0}}{2 \pi} f^{2} \nabla \theta,
$$

where $L$ is penetration depth tensor. When $X, Y, Z$ are principal axes of the superconductor, it has components

$$
L=\left(\begin{array}{ccc}
\Lambda_{X}^{2} & 0 & 0 \\
0 & \Lambda_{Y}^{2} & 0 \\
0 & 0 & \Lambda_{Z}^{2}
\end{array}\right) .
$$

Following Clem [8], we look for a solution of the form

$$
\mathbf{A}=\mathbf{A}_{\text {bulk }}+\mathbf{A}_{\text {surface }} \text {. }
$$

$\mathbf{A}_{\text {bulk }}$ is the solution for a single vortex in an infinitely thick specimen and consequently has no $Z$ dependence. $\mathbf{A}_{\text {surface }}$ is the general solution with the correct boundary conditions but with the right-hand side of the Ginzburg-Landau equation set to zero.

Clem [14] solved for the bulk term in the isotropic case by using an order parameter for a single vortex of the form

$$
\Psi=f e^{i \theta}=\frac{\rho}{\sqrt{\rho^{2}+\xi^{2}}} e^{-i \phi},
$$

where $\rho$ is the radius from the axis of the vortex, $\phi$ is the azimuthal angle, and $\xi$ is the coherence length. Klemm and Clem $[9,11]$ later extended this solution to the anisotropic case so that the order parameter becomes

$$
\Psi=\sqrt{\frac{X^{2} / \Lambda_{Y}^{2}+Y^{2} / \Lambda_{X}^{2}}{X^{2} / \Lambda_{Y}^{2}+Y^{2} / \Lambda_{X}^{2}+\xi^{2} / \Lambda^{2}}} e^{-i \arg \left(X / \Lambda_{Y}+i Y / \Lambda_{X}\right)},
$$

where $\Lambda=\left(\Lambda_{X} \Lambda_{Y} \Lambda_{Z}\right)^{1 / 3}$. This gives the magnetic flux density as

$$
\mathbf{B}=\frac{\Phi_{0}}{2 \pi \Lambda_{X} \Lambda_{Y}} \frac{K_{0}(R)}{(\xi / \Lambda) K_{1}(\xi / \Lambda)} \widehat{\mathbf{Z}},
$$

where $R=\sqrt{\left(X / \Lambda_{Y}\right)^{2}+\left(Y / \Lambda_{X}\right)^{2}+(\xi / \Lambda)^{2}}$ and $K_{0}$ and $K_{1}$ are zero and first-order modified Bessel functions.

Fourier transforming the flux density gives

$$
\widetilde{\mathbf{B}}=\frac{\Phi_{0} K_{1}(Q \xi / \Lambda)}{Q K_{1}(\xi / \Lambda)} \widehat{\mathbf{Z}}
$$

where $Q=\sqrt{1+k_{X}^{2} \Lambda_{Y}^{2}+k_{Y}^{2} \Lambda_{X}^{2}}$. Since $\mathbf{B}=\nabla \times \mathbf{A}$, in Fourier space, we have $\widetilde{\mathbf{B}}=\left(i k_{X}{ }_{X} \widetilde{A}_{Y}-i k_{Y} \widetilde{A}_{X}\right) \widehat{\mathbf{Z}}=i \mathbf{k}_{\perp} \times \widetilde{\mathbf{A}}$ where $\mathbf{k}_{\perp}=\left(k_{X}, k_{Y}, 0\right)$. Imposing the additional requirement that $\mathbf{A}$ obey the London gauge $\nabla \cdot \mathbf{A}=0$ so that $i k_{X} \widetilde{A}_{X}+$ $i k_{Y} \widetilde{A}_{Y}=0$, the vector potential in Fourier space is

$$
\widetilde{\mathbf{A}}_{\text {bulk }}=\frac{i \Phi_{0} K_{1}(Q \xi / \Lambda)}{k_{\perp}^{2} Q K_{1}(\xi / \Lambda)}\left(\begin{array}{c}
k_{Y} \\
-k_{X} \\
0
\end{array}\right) .
$$

We now find the surface term, $\mathbf{A}_{\text {surface }}$. This is the general solution to the Ginzburg-Landau equation but where the right-hand side is set equal to zero. We use the approximation introduced by Clem [8] that the surface term should be only weakly influenced by the vortex core and so set $f^{2}=1$ in the search for a solution. The validity of this simplification was confirmed by Brandt [15] who modeled the complete vortex and found that the core only expands by a few percent as it approaches the surface of the superconductor. The surface term is thus the solution to

$$
\mathbf{A}+L \nabla \times(\nabla \times \mathbf{A})=0 .
$$

It should be noted that setting the right-hand side of the Ginzburg-Landau equation [Eq. (2)] to zero fixes the gauge of the vector potential and for an anisotropic superconductor, this is not the London gauge. Thus we cannot say $\nabla \cdot \mathbf{A}=0$ nor make the convenient replacement $\nabla \times(\nabla \times \mathbf{A})=-\nabla^{2} \mathbf{A}$. Instead, we must deal with the awkward cross terms arising from the double curl.

Taking the Fourier transform of Eq. (10) gives

$$
\widetilde{\mathbf{A}}+L\left(i \mathbf{k}_{\perp}+\widehat{\mathbf{Z}} \partial_{z}\right) \times\left(\left(i \mathbf{k}_{\perp}+\widehat{\mathbf{Z}} \partial_{z}\right) \times \widetilde{\mathbf{A}}\right)=0 .
$$

We now postulate a solution of the form $\widetilde{\mathbf{A}}\left(k_{X}, k_{Y}, Z\right)=$ $\mathbf{a}\left(k_{X}, k_{Y}\right) e^{\beta Z}$ and the resulting equation can be written in matrix form as

$$
\begin{aligned}
& \left(\begin{array}{ccc}
1+k_{Y}^{2} \Lambda_{X}^{2}-\Lambda_{X}^{2} \beta^{2} & -k_{X} k_{Y} \Lambda_{X}^{2} & i k_{X} \Lambda_{X}^{2} \beta \\
-k_{X} k_{Y} \Lambda_{Y}^{2} & 1+k_{X}^{2} \Lambda_{Y}^{2}-\Lambda_{Y}^{2} \beta^{2} & i k_{Y} \Lambda_{Y}^{2} \beta \\
i k_{X} \Lambda_{Z}^{2} \beta & i k_{Y} \Lambda_{Z}^{2} \beta & 1+\Lambda_{Z}^{2}\left(k_{X}^{2}+k_{Y}^{2}\right)
\end{array}\right) \mathbf{a} \\
& =\left(\begin{array}{l}
0 \\
0 \\
0
\end{array}\right) .
\end{aligned}
$$


The above equation gives nonzero solutions for the vector potential if the matrix cannot be inverted. To achieve this, values of $\beta$ must be found to make the determinant zero. At this point, we introduce the symmetry of the problem otherwise the answers become very lengthy. For this experiment, the specimen was tilted about the $a$ axis and thus, $X\|c, Y\| a$, and $Z \| b$, so that $\Lambda_{X}=\Lambda_{c}, \Lambda_{Y}=\Lambda_{a b}$, and $\Lambda_{Z}=\Lambda_{a b}$. There are then four possible values of $\beta$ :

$$
\beta_{1,3}= \pm Q_{a} / \Lambda_{a b}
$$

and

$$
\beta_{2,4}= \pm Q / \Lambda_{c}
$$

with corresponding eigenvectors

$$
\mathbf{a}_{1,3}=\left(\begin{array}{c}
0 \\
\pm i Q_{a} /\left(k_{Y} \Lambda_{a b}\right) \\
1
\end{array}\right)
$$

and

$$
\mathbf{a}_{2,4}=\left(\begin{array}{c} 
\pm i \Lambda_{c}\left(1+k_{X}^{2} \Lambda_{a b}^{2}\right) /\left(\Lambda_{a b}^{2} k_{X} Q\right) \\
\pm i \Lambda_{c} k_{Y} / Q \\
1
\end{array}\right)
$$

where $\quad Q_{a}=\sqrt{1+\left(k_{X}^{2}+k_{Y}^{2}\right) \Lambda_{a b}^{2}} \quad$ and $\quad Q=$ $\sqrt{1+k_{X}^{2} \Lambda_{a b}+k_{Y}^{2} \Lambda_{c}}$. The complete vector potential inside the superconductor is then

$$
\widetilde{\mathbf{A}}_{\text {inside }}=\widetilde{\mathbf{A}}_{\text {bulk }}+\sum_{n=1}^{4} c_{n} \mathbf{a}_{n} e^{\beta_{n} z},
$$

where $c_{1}-c_{4}$ need to be determined by the boundary conditions.

This leaves the vector potential outside the superconductor to be determined. Maxwell's third equation gives $\nabla \times \mathbf{B}=0$ as there are no electrical currents outside the superconductor so the vector potential obeys $\nabla \times(\nabla \times \mathbf{A})=0$. This time, the London gauge, $\nabla \cdot \mathbf{A}=0$, may safely be invoked to give

$$
\nabla^{2} \mathbf{A}_{\text {outside }}=0
$$

or, in Fourier space,

$$
-k_{\perp}^{2} \tilde{\mathbf{A}}+\frac{\partial^{2} \widetilde{\mathbf{A}}}{\partial Z^{2}}=0 .
$$

The solution to this is

$$
\begin{aligned}
\tilde{\mathbf{A}}_{\text {top }} & =\left(\begin{array}{c}
c_{5} \\
c_{6} \\
i\left(c_{5} k_{X}+c_{6} k_{Y}\right) / k_{\perp}
\end{array}\right) e^{-k_{\perp} Z}, \\
\tilde{\mathbf{A}}_{\text {bottom }} & =\left(\begin{array}{c}
c_{7} \\
c_{8} \\
-i\left(c_{7} k_{X}+c_{8} k_{Y}\right) / k_{\perp}
\end{array}\right) e^{k_{\perp} Z},
\end{aligned}
$$

where the $Z$ components are determined by the London gauge. We can now simplify the equations as symmetry requires that $A_{X, Y}(-Z)=A_{X, Y}(Z)$ and $A_{Z}(-Z)=-A_{Z}(Z)$. This gives $c_{1}=-c_{3}, c_{2}=-c_{4}, c_{5}=c_{7}$, and $c_{6}=c_{8}$.
Summarizing, so far, the vector potential inside the superconductor is

$$
\begin{aligned}
\widetilde{\mathbf{A}}_{\text {inside }}= & \widetilde{\mathbf{A}}_{\text {bulk }}+2 c_{1}\left(\begin{array}{l}
a_{1, X} \cosh \left(\beta_{1} Z\right) \\
a_{1, Y} \cosh \left(\beta_{1} Z\right) \\
a_{1, Z} \sinh \left(\beta_{1} Z\right)
\end{array}\right) \\
& +2 c_{2}\left(\begin{array}{c}
a_{2, X} \cosh \left(\beta_{2} Z\right) \\
a_{2, Y} \cosh \left(\beta_{2} Z\right) \\
a_{2, Z} \sinh \left(\beta_{2} Z\right)
\end{array}\right),
\end{aligned}
$$

and above and below the superconductor, the vector potential is

$$
\widetilde{\mathbf{A}}_{\text {above, below }}=\left(\begin{array}{c}
c_{5} \\
c_{6} \\
\pm i\left(c_{5} k_{X}+c_{6} k_{Y}\right) / k_{\perp}
\end{array}\right) e^{\mp k_{\perp} Z}
$$

To fix the values of $c_{1}, c_{2}, c_{5}$, and $c_{6}$, we invoke the following boundary conditions. (1) In order to calculate the phase shift from the vector potential, the $X$ and $Y$ components of the vector potential must change continuously across the boundary between the superconductor and vacuum at $Z=$ $\pm d$. (2) The in-plane flux density $B_{\|}$must be continuous across the boundaries at $Z= \pm d$ as there are no currents confined to the surface of the superconductor. There is also the requirement that the normal component of the flux density $B_{\perp}$ be continuous but this arises from Maxwell's third equation, $\nabla \cdot \mathbf{B}=0$, and by using a vector potential, it is automatically satisfied.

The condition (1) that the in-plane vector potential is continuous at $Z= \pm d$ gives two equations (one for each component):

$$
\widetilde{A}_{\text {bulk }, X}+2 c_{1} a_{1, X} \cosh \left(\beta_{1} d\right)+2 c_{2} a_{2, X} \cosh \left(\beta_{2} d\right)=c_{5} e^{-k_{\perp} d},
$$

$$
\widetilde{A}_{\text {bulk }, Y}+2 c_{1} a_{1, Y} \cosh \left(\beta_{1} d\right)+2 c_{2} a_{2, Y} \cosh \left(\beta_{2} d\right)=c_{6} e^{-k_{\perp} d} .
$$

Calculating the flux density via $\mathbf{B}=\nabla \times \mathbf{A}$ or, in Fourier space, $\widetilde{\mathbf{B}}=\left(i \mathbf{k}_{\perp}+\widehat{\mathbf{z}} \partial_{z}\right) \times \widetilde{\mathbf{A}}$ and matching its in-plane components at the interface gives two more:

$$
\begin{aligned}
& \left(-\left(k_{Y} / k_{\perp}\right)\left(c_{5} k_{X}+c_{6} k_{Y}\right)+c_{6} k_{\perp}\right) e^{-k_{\perp} d} \\
& \quad=2 c_{1} D_{1} \sinh \left(\beta_{1} d\right)+2 c_{2} D_{2} \sinh \left(\beta_{2} d\right), \\
& \left(-c_{5} k_{\perp}+\left(k_{X} / k_{\perp}\right)\left(c_{5} k_{X}+c_{6} k_{Y}\right)\right) e^{-k_{\perp} d} \\
& \quad=2 c_{1} D_{3} \sinh \left(\beta_{1} d\right)+2 c_{2} D_{4} \sinh \left(\beta_{2} d\right),
\end{aligned}
$$

where $D_{1}=i k_{Y} a_{1, Z}-\beta_{1} a_{1, Y}, \quad D_{2}=i k_{Y} a_{2, Z}-\beta_{2} a_{2, Y}, \quad D_{3}=$ $\beta_{1} a_{1, X}-i k_{X} a_{1, Z}$, and $D_{4}=\beta_{2} a_{2, X}-i k_{X} a_{2, Z}$. 
Writing these four equations in matrix form gives

$$
\left(\begin{array}{cccc}
2 D_{1} \sinh \left(\beta_{1} d\right) & 2 D_{2} \sinh \left(\beta_{2} d\right) & \frac{k_{X} k_{Y}}{k_{\perp}} e^{-k_{\perp} d} & \left(\frac{k_{Y}^{2}}{k_{\perp}}-k_{\perp}\right) e^{-k_{\perp} d} \\
2 D_{3} \sinh \left(\beta_{1} d\right) & 2 D_{4} \sinh \left(\beta_{2} d\right) & -\left(\frac{k_{X}}{k_{\perp}}-k_{\perp}\right) e^{-k_{\perp} d} & -\frac{k_{X} k_{Y}}{k_{\perp}} e^{-k_{\perp} d} \\
2 a_{1, x} \cosh \left(\beta_{1} d\right) & 2 a_{2, x} \cosh \left(\beta_{2} d\right) & -e^{-k_{\perp} d} & 0 \\
2 a_{1, y} \cosh \left(\beta_{1} d\right) & 2 a_{2, y} \cosh \left(\beta_{2} d\right) & 0 & -e^{-k_{\perp} d}
\end{array}\right)\left(\begin{array}{l}
c_{1} \\
c_{2} \\
c_{5} \\
c_{6}
\end{array}\right)=\left(\begin{array}{c}
0 \\
0 \\
-\widetilde{A}_{\text {bulk,X}} \\
-\widetilde{A}_{\text {bulk,Y}}
\end{array}\right)
$$

The coefficients $c_{1}, c_{2}, c_{5}, c_{6}$ can then be found by inverting the matrix, and then after substituting the answers into Eqs. (22) and (23), the vector potential is fully determined. It should be noted that although we use Klemm and Clem's solution for $\mathbf{A}_{\text {bulk }}$, Eq. (28) shows that our method has the convenient feature that any model for $\mathbf{A}_{\text {bulk }}$ could be used with equal facility.

\section{Phase shift}

The magnetic contribution to the phase shift suffered by the electron beam after passing through a specimen is related to the vector potential via the Aharanov-Bohm expression:

$$
\phi(x, y)=-\frac{2 \pi e}{h} \int_{-\infty}^{\infty} \mathbf{A}(x, y, z) \cdot d \mathbf{l}
$$

where $d \mathbf{l}$ is an increment along the trajectory of the electrons shown in Fig. 3. If the unit vectors in $x, y$, and $z$ of the microscope coordinate system are denoted $\mathbf{i}, \mathbf{j}$, and $\mathbf{k}$ and those in $X, Y$, and $Z$ of the specimen coordinate system are denoted $\mathbf{I}, \mathbf{J}$, and $\mathbf{K}$, the two sets of unit vectors are related via

$$
\begin{gathered}
\mathbf{I}=\mathbf{i} \cos \alpha+\mathbf{k} \sin \alpha, \\
\mathbf{J}=-\mathbf{j}, \\
\mathbf{K}=\mathbf{i} \sin \alpha-\mathbf{k} \cos \alpha .
\end{gathered}
$$

If the electron passes through the specimen at position $(X, Y, 0)$ and if for the preceding part of its journey we label the height above the specimen in the $Z$ direction $w$ (see Fig. 3), its position at any point in its trajectory is given by

$$
\mathbf{l}=(X-w \tan \alpha) \mathbf{I}+Y \mathbf{J}+w \mathbf{K} .
$$

By differentiating the above equation, an increment in its trajectory $d \mathbf{l}$ can be related to an increment in $w$ via

$$
d \mathbf{l}=(-\tan \alpha \mathbf{I}+\mathbf{K}) d w .
$$

The phase shift written in terms of the specimen coordinates is thus

$$
\begin{aligned}
\phi(X, Y)= & -\frac{2 \pi e}{h} \int_{-\infty}^{\infty} \mathbf{A}(X-w \tan \alpha, Y, w) \\
& \cdot\left(\begin{array}{c}
-\tan \alpha \\
0 \\
1
\end{array}\right) d w .
\end{aligned}
$$

In the previous section, the vector potential was calculated in Fourier space. This is related to the vector potential in real-space via the inverse Fourier transform

$$
\begin{aligned}
& \mathbf{A}(X, Y, Z) \\
& \quad=\frac{1}{4 \pi^{2}} \int_{-\infty}^{\infty} \int_{-\infty}^{\infty} \widetilde{\mathbf{A}}\left(k_{X}, k_{Y}, Z\right) e^{i \mathbf{k}_{\perp} \cdot \mathbf{R}} d k_{X} d k_{Y},
\end{aligned}
$$

where $\mathbf{R}=(X, Y, Z)$. Thus it follows that

$$
\begin{aligned}
& \mathbf{A}(X-w \tan \alpha, Y, Z) \\
& \quad=\frac{1}{4 \pi^{2}} \iint \widetilde{\mathbf{A}}\left(k_{X}, k_{Y}, Z\right) e^{i \mathbf{k}_{\perp} \cdot \mathbf{R}} e^{-i k_{X} w \tan \alpha} d k_{X} d k_{Y} .
\end{aligned}
$$

So the phase shift is

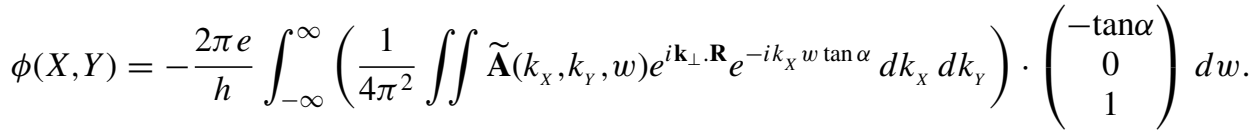

It can be seen from Fig. 3 that if an electron passes through a point $(X, Y, 0)$ on the specimen, it passes through a point $(x, y, 0)$ in the $x y$ plane where $x=X \cos \alpha$ and $y=-Y$. The phase shift in the $x y$ plane (which is equivalent to the plane in which the image is taken) is now found by making this substitution:

$$
\phi(x, y)=-\frac{2 \pi e}{h} \int_{-\infty}^{\infty}\left(\frac{1}{4 \pi^{2}} \iint \widetilde{\mathbf{A}}\left(k_{X}, k_{Y}, w\right) e^{i k_{X} x / \cos \alpha} e^{-i k_{Y} y} e^{-i k_{X} w \tan \alpha} d k_{X} d k_{Y}\right) \cdot\left(\begin{array}{c}
-\tan \alpha \\
0 \\
1
\end{array}\right) d w .
$$

Now, let $k_{x}=k_{X} / \cos \alpha$ and $k_{y}=-k_{Y}$ :

$$
\phi(x, y)=\frac{2 \pi e}{h} \int_{-\infty}^{\infty}\left(\frac{1}{4 \pi^{2}} \iint \widetilde{\mathbf{A}}\left(k_{x} \cos \alpha,-k_{y}, w\right) e^{i k_{x} x} e^{i k_{y} y} e^{-i k_{x} w \sin \alpha} \cos \alpha d k_{x} d k_{y}\right) \cdot\left(\begin{array}{c}
-\tan \alpha \\
0 \\
1
\end{array}\right) d w
$$


Changing the order of integration gives

$$
\phi(x, y)=\frac{2 \pi e \cos \alpha}{h} \frac{1}{4 \pi^{2}} \iint e^{i \mathbf{k}_{\perp} \cdot \mathbf{r}}\left(\int_{-\infty}^{\infty} \widetilde{\mathbf{A}}\left(k_{x} \cos \alpha,-k_{y}, w\right) e^{-i k_{x} w \sin \alpha} d w\right) d k_{x} d k_{y} \cdot\left(\begin{array}{c}
-\tan \alpha \\
0 \\
1
\end{array}\right),
$$

or representing an inverse Fourier transform as IFT and using the flux quantum $\Phi_{0}$, we have

$$
\phi(x, y)=\operatorname{IFT}\left[\frac{\pi}{\Phi_{0}}\left(\begin{array}{c}
-\sin \alpha \\
0 \\
\cos \alpha
\end{array}\right) \cdot \int_{-\infty}^{\infty} \widetilde{\mathbf{A}}\left(k_{x} \cos \alpha,-k_{y}, w\right) e^{-i k_{x} w \sin \alpha} d w\right] .
$$

The integral over $w$ is straightforward as it only involves exponential functions but it is very lengthy so it and the scalar product were performed symbolically using MATLAB. Only the final inverse Fourier transform, which gives the phase, was evaluated numerically.

A method to check the correctness of the solution is to plot the phase shift as a contour map for $\alpha=90^{\circ}$. This gives the $B$ field projected through the thickness of the specimen and is shown in Fig. 4. It can be seen that the correct boundary conditions are fulfilled: the field lines spread as they approach the specimen surface from the interior, and outside the specimen they are straight so that the field resembles that from a monopole if viewed far from the vortex. The figure shows that the effect of increasing the coherence length $\xi_{V}$ is to make the field less intense near the center of the vortex as expected.

\section{Image simulation}

Once the phase shift $\phi(x, y)$ has been calculated, the wave function of the electron beam is $\psi_{0}(x, y)=e^{i \phi}$ and the intensity of the in-focus bright-field image is $I_{0}(x, y)=$ $\left|\psi_{0}(x, y)\right|^{2}$. It is immediately clear that this gives 1 and an in-focus image is therefore featureless. In order to visualize flux vortices, out-of-focus images must be taken. Taking an out-of-focus image is equivalent to propagating the wave function through free space by a distance $\Delta f$, known as the defocus. This is done via the Fresnel-Kirchoff integral [7] so that the defocused wave function $\psi_{\Delta f}(x, y)$ is related to the in-focus wave function via

$$
\begin{aligned}
\psi_{\Delta f}(x, y)= & \frac{1}{\lambda \Delta f} \iint \psi_{0}\left(x^{\prime}, y^{\prime}\right) \\
& \times e^{\frac{i \pi}{\lambda \Delta f}\left(\left(x-x^{\prime}\right)^{2}+\left(y-y^{\prime}\right)^{2}\right)} d x^{\prime} d y^{\prime},
\end{aligned}
$$

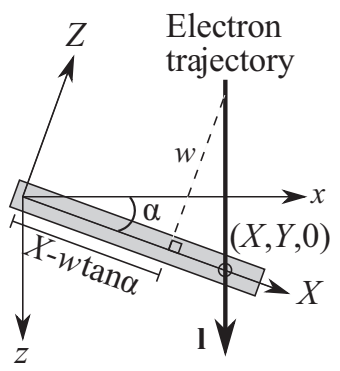

FIG. 3. The relationship between the coordinates $X, Y, Z$, referring to the specimen (shown by the grey rectangle) and the microscope coordinates $x, y, z$. where $\lambda$ is the electron wavelength. This is a convolution and so is more conveniently evaluated as a multiplication in Fourier space via

$$
\widetilde{\psi}_{\Delta f}=\widetilde{\psi}_{0} e^{-i \lambda \Delta f k^{2} / 4 \pi} .
$$

After inverse transforming, the intensity in the out-of-focus image is given by $I_{\Delta f}=\left|\psi_{\Delta f}\right|^{2}$.

\section{EXPERIMENTAL METHOD}

$\mathrm{MgB}_{2}$ single crystals were synthesized by the peritectic decomposition of $\mathrm{MgNB}_{9}$ and their quality and bulk properties have been well characterized by a variety of experimental techniques [16-18]. The samples were prepared for electron microscopy at the Technical University of Denmark (DTU) using a Helios Nanolab focused ion beam microscope (FIB). This is a dual-beam instrument in which a beam of gallium ions is used to mill the specimen whilst secondary electrons emitted by the specimen are used to produce an image, however, an electron beam can also be used to illuminate the specimen in order to take images without damaging the specimen.

The $\mathrm{MgB}_{2}$ single crystals were about $1 \times 1 \mathrm{~mm}^{2}$ in the $a b$ plane and about $100-\mu \mathrm{m}$ thick in the $c$ direction. The in situ lift-out technique was used to prepare the sample for electron microscopy. First, the FIB was used to deposit a 3- $\mu \mathrm{m}$ thick, $30 \times 5 \mu \mathrm{m}^{2}$ rectangle of platinum onto the $a b$ surface to protect the sample beneath from ion damage. Trenches were then milled to a depth of $20 \mu \mathrm{m}$ in the $c$ direction around this (a)

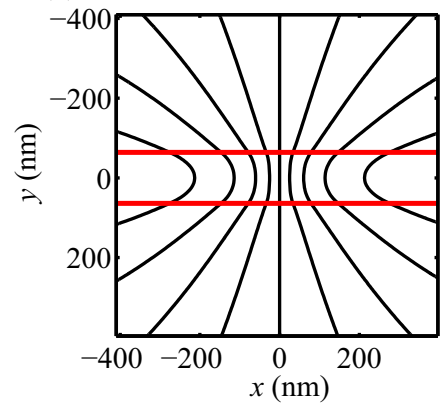

(b)

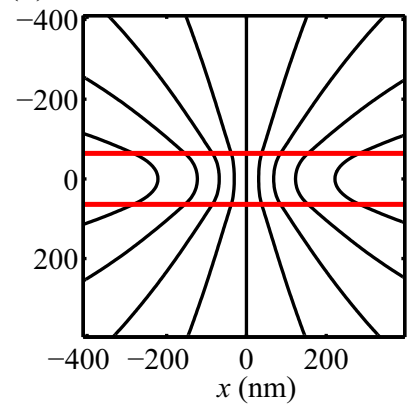

FIG. 4. (Color online) Contours of the phase shift spaced by 0.2 rad illustrating the projected $B$ field from a flux vortex with $\Lambda_{a b}=100 \mathrm{~nm}$ and $\Lambda_{c}=120 \mathrm{~nm}$ and (a) $\xi_{V}=1 \mathrm{~nm}$ and (b) $\xi_{V}=36$ $\mathrm{nm}$. The red lines indicate the surface of the specimen, which is 128-nm thick. The $a$ axis is parallel to $x$ with $c$ pointing into the page. 
to produce a slab standing in the center of a crater. The top surface of the sample was smooth and this avoided the creation of the longitudinal thickness undulations reported in our last paper [4].

A movable needle known as a micromanipulator was attached to the slab using platinum deposition and the slab was cut away from the rest of the specimen and extracted on the end of the micromanipulator. A sample cannot be tilted more than $25^{\circ}$ in the electron microscope so to achieve a higher tilt angle, the FIB was used to cut a slot at $45^{\circ}$ to the plane of an "Omniprobe" grid. Further platinum deposition was used to attach the sample to this slot and the micromanipulator was then cut away, leaving the sample attached to the grid and tilted about its $a$ axis by $45^{\circ}$ with respect to the plane of the grid. The sample was then thinned to approximately $200 \mathrm{~nm}$ so that it was electron transparent using a $30 \mathrm{kV}$ Ga ion beam. Finally, the specimen surfaces were polished by a low-energy $(2 \mathrm{kV}) \mathrm{Ga}$ ion beam to minimize the damage layer caused by FIB milling.

Electron microscopy was undertaken at DTU using an FEI Titan 80-300ST transmission electron microscope operated at $300 \mathrm{kV}$ equipped with a Gatan imaging filter to record images. Under normal operating conditions, the main objective lens of the microscope applies a 2-T field to the specimen, so to avoid this, the microscope was operated in low-magnification mode with the main objective lens set to a low value and the image was focused with the diffraction lens. Prior to imaging vortices, electron diffraction was used to make a fine adjustment of a few degrees so that the tilt was purely about the $a$ axis. Adjusting the sample so that it is tilted purely about $a$ can be performed to better than $0.5^{\circ}$ but this may alter the overall tilt angle and we judge that the tilt angle was $\alpha=45 \pm 5^{\circ}$.

The simulations were based on elastic electron scattering so experimental images were energy filtered so that only electrons that had lost $0-10 \mathrm{eV}$ on passing through the specimen contributed and an aperture was used so that only the 000 beam and the low-angle scattering from the vortices contributed to the image and the other crystallographic beams were excluded. The sample was cooled using a Gatan liquid-helium cooled "IKHCHDT3010-Special" tilt-rotate holder, which has a base temperature of $10 \mathrm{~K}$.

The defocus and magnification were calibrated by acquiring images with the same lens settings as the original images from Agar Scientific's "S106" calibration specimen, which consists of lines spaced by $463 \mathrm{~nm}$ ruled on an amorphous film. The defocus was found by taking digital Fourier transforms of the images acquired from the calibration specimen and measuring the radii of the dark rings that result from the contrast transfer function [19].

A thickness map of the specimen was created by dividing an unfiltered image by an energy-filtered image and taking the natural logarithm [20], which gives the thickness parallel to the electron beam, $l$, as a multiple of the inelastic mean free path $\lambda_{i}$. To determine $\lambda_{i}$, an electron hologram was taken at room temperature at an edge of the specimen, which gives a phase shift proportional to the thickness, $\phi=C_{E} V_{0} l . C_{E}$ is a constant that depends only on the microscope voltage and has the value $6.523 \times 10^{6} \mathrm{~m}^{-1} \mathrm{~V}^{-1}$ at $300 \mathrm{kV}$. The mean inner potential $V_{0}$ was calculated as $V_{0}=17.71 \mathrm{~V}$ from the theoretical scattering factors given in Ref. [21], giving $\lambda_{i}=244 \pm 5 \mathrm{~nm}$, and the
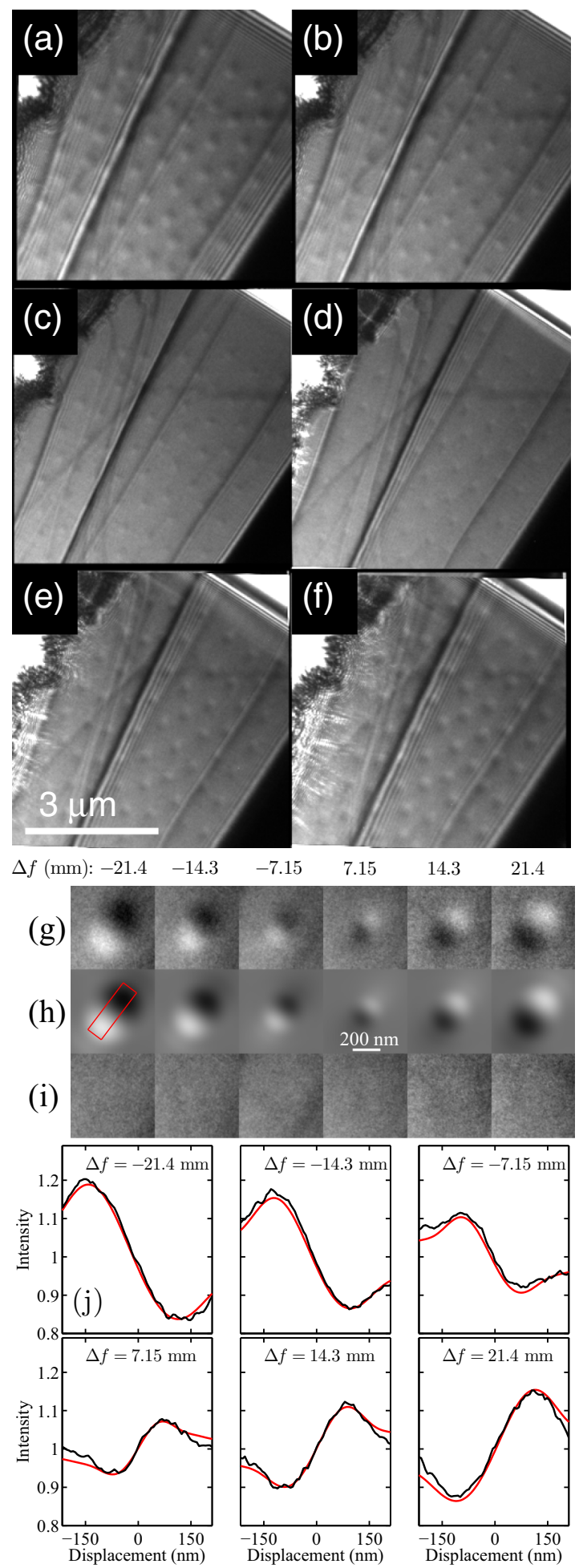

FIG. 5. (Color online) (a)-(f) Defocus series showing flux vortices in $\mathrm{MgB}_{2}$ at $10.8 \mathrm{~K}$ in a field of $4.8 \mathrm{mT}$ with defoci: (a) $\Delta f=$ -21.4 , (b) -14.3 , (c) -7.15 , (d) 7.15, (e) 14.3, and (f) $21.4 \mathrm{~mm}$. (g) Average vortex images at each defocus level. (h) Average simulated images. (i) Difference images between experiment and simulation. (j) Line scans across the images from the region shown by the red box in (h). Black lines show experimental data and red lines the fit.

thickness $l$ was varied from 200-290 nm across the field of view of Fig. 5. Ideally, the thickness of the whole specimen 
would have been determined by electron holography but the field of view of the interference region was not sufficiently large.

A simplex algorithm [22] was used to minimize the reduced $\chi^{2}$ value between the experimental images and simulations by fitting the vortex positions and the in-plane rotation angle of the vortices as well as $\Lambda_{a b}, \Lambda_{c}$, and $\xi_{V}$. The reduced $\chi^{2}$ value is defined as $\chi^{2} \equiv(1 / N) \sum_{j=1}^{N}\left(I_{j}^{\text {experiment }}-I_{j}^{\text {simulation }}\right)^{2} / \sigma_{j}^{2}$, where $N$ is the number of pixels used in the fit, $I_{j}$ is the intensity of pixel $j$ in the image, and $\sigma_{j}$ is the noise associated with pixel $j$. We used $\sigma_{j}^{2}=c I_{j}^{\text {simulation }}$ having previously taken a series of images of the vacuum with different electron intensities. A graph of the standard deviation versus the average intensity showed the noise was shot noise (so that the square of the noise was proportional to the image intensity) and gave the proportionality constant $c$ relating the counts recorded on the detector to the number of electrons received. Unlike our previous publication where separate fits were made for each vortex [23], here, all the vortex images were fit simultaneously with a single value of $\Lambda_{a b}, \Lambda_{c}$, and $\xi_{V}$ using the model described in Sec. II.

\section{RESULTS}

Figures 5(a)-5(f) show an experimental defocus series acquired at $10.8 \mathrm{~K}$ (the base temperature of our cooling stage) in a field of $4.8 \mathrm{mT}$. Sixty-eight images of vortices that were the least affected by bend contrast were fit with a single value of $\Lambda_{a b}, \Lambda_{c}$, and $\xi_{V}$ along with the position of each vortex and its in-plane rotation angle using a simplex algorithm [22] to minimize the reduced $\chi^{2}$ value. The specimen thickness and tilt angle were fixed at their calibrated values.

Figure $5(\mathrm{~g})$ shows the average of these images at each defocus level and (h) shows the average of the fitted simulations. To demonstrate that the fit is good, (i) shows the difference between images and simulations and (j) compares line scans taken across the vortex images.

$\Lambda_{a b}, \Lambda_{c}$, and $\xi_{V}$ were then altered in turn and the error bar on each was judged by the point at which the difference images displayed a discernibly worse fit as shown in Figs. 6(a)$6(d)$. This corresponded to an increase in the reduced $\chi^{2}$ of 0.009 and the variation of $\chi^{2}$ as each parameter is varied, as shown in Fig. 6(e). This yielded $\Lambda_{a b}=107 \pm 33 \mathrm{~nm}$, $\Lambda_{c}=134 \pm 6 \mathrm{~nm}$, and $\xi_{V}=31 \pm 9 \mathrm{~nm}$. The images are much more sensitive to the value of $\Lambda_{c}$ than to $\Lambda_{a b}$ as a consequence of mounting the sample tilted about the $a$ axis; had it been tilted about $c$, the errors on $\Lambda_{a b}$ and $\Lambda_{c}$ would be reversed. In a previous paper [4], we obtained the more precise value of $\Lambda_{a b}=107 \pm 8 \mathrm{~nm}$ using a sample cut in the $a b$ plane.

To check for amorphous "dead layers" of nonsuperconducting material on the sample surfaces caused by ion thinning, the thickness of the crystalline component of the sample was measured using the convergent beam diffraction technique described in Ref. [19]. This showed no difference between the total and crystalline thicknesses to within the experimental error of $\pm 10 \mathrm{~nm}$. Reducing the calibrated thickness values by $50 \mathrm{~nm}$ to account for the largest conceivable dead layer, reduced $\Lambda_{a b}$ by $13 \mathrm{~nm}$, reduced $\Lambda_{c}$ by $27 \mathrm{~nm}$, and increased
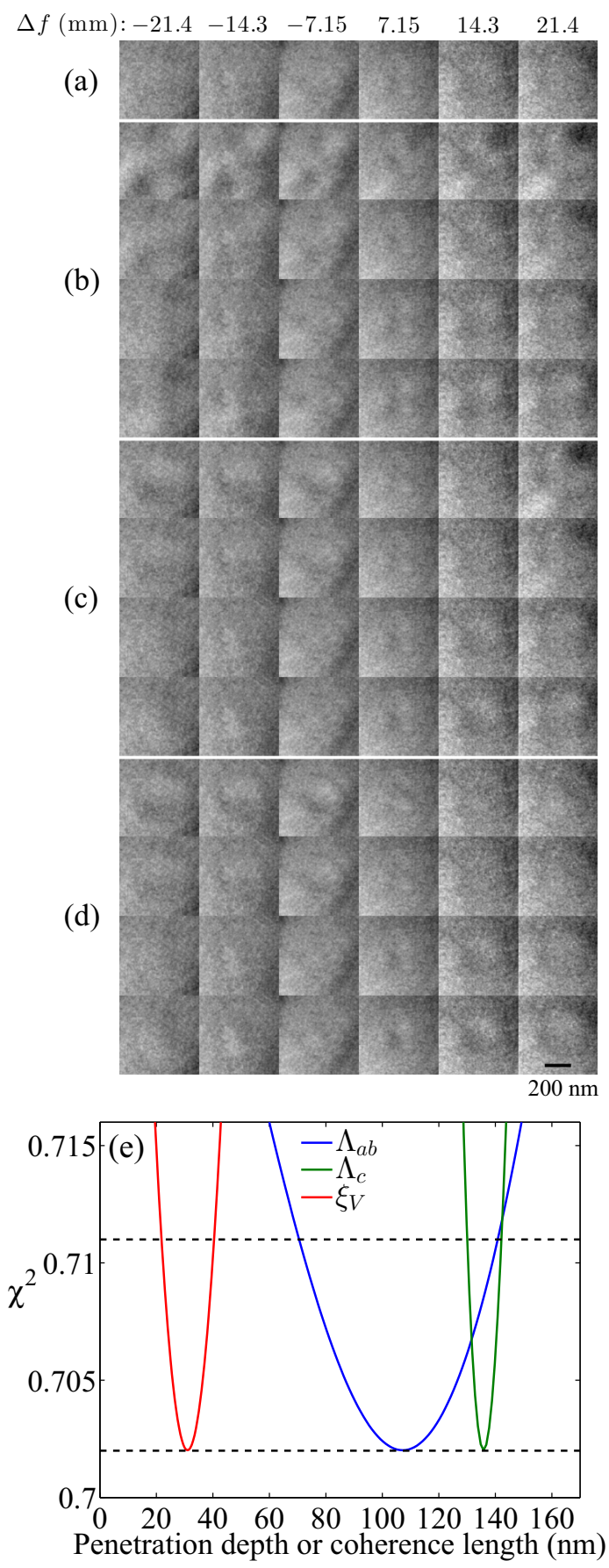

FIG. 6. (Color online) Difference images between the average defocus series and simulated images as each parameter is varied. (a) Difference images for the best-fit values of the parameters. (b) Difference images when $\Lambda_{a b}$ is varied: the top row shows difference images when $\Lambda_{a b}$ is set at two error bars below the best-fit value. The next row is for $\Lambda_{a b}$ set one error bar below. The next two rows are for $\Lambda_{a b}$ increased one and two error bars above the best-fit value, respectively. (c) A similar series showing the effect of changing $\Lambda_{c}$. (d) Series showing the effect of changing $\xi_{V}$. (e) Reduced $\chi^{2}$ values as $\Lambda_{a b}, \Lambda_{c}$, and $\xi_{V}$ are adjusted. Acceptable values of $\chi^{2}$ lie between the dashed lines.

$\xi_{V}$ by $10 \mathrm{~nm}$ without altering the quality of the fit. Taking this into account gives $\Lambda_{a b}=100 \pm 35 \mathrm{~nm}, \Lambda_{c}=120 \pm 15 \mathrm{~nm}$, 
and $\xi_{V}=36 \pm 10 \mathrm{~nm}$. The anisotropy ratio in the penetration depth is then $\gamma_{\Lambda}=1.2 \pm 0.4$ and the coherence lengths are $\xi_{a b}=\gamma^{2 / 3} \xi_{V}=41 \pm 13 \mathrm{~nm}$ and $\xi_{c}=\gamma^{-1 / 3} \xi_{V}=34 \pm$ $10 \mathrm{~nm}$. Alternatively, using the more precise value of $\Lambda_{a b}=$ $107 \pm 8 \mathrm{~nm}$ and $\Lambda_{c}=120 \pm 15 \mathrm{~nm}$ gives $\gamma_{\Lambda}=1.12 \pm 0.16$, $\xi_{a b}=39 \pm 11 \mathrm{~nm}$, and $\xi_{c}=35 \pm 10 \mathrm{~nm}$.

\section{DISCUSSION}

We now compare the values obtained here with those found using other techniques. These show that the conditions used $(4.8 \mathrm{mT}$ and $10.8 \mathrm{~K})$ were in the low-field limit $(<100 \mathrm{mT}$ as established by neutron diffraction) but not quite in the lowtemperature limit ( $<5 \mathrm{~K}$ from radio-frequency measurements). In 2005, Fletcher et al. [24] performed radio-frequency measurements, which gave the change in the low-field penetration depth with temperature but not absolute values. At $10.8 \mathrm{~K}, \Lambda_{a b}$ increased by $12 \pm 1 \mathrm{~nm}$, and $\Lambda_{c}$ increased by $18 \pm 4 \mathrm{~nm}$ with respect to their low-temperature values. Subtracting these from our most precise measurements of the penetration depths gives $\Lambda_{a b}=95 \pm 8 \mathrm{~nm}$ and $\Lambda_{c}=102 \pm 15 \mathrm{~nm}$ and the anisotropy as $\gamma=1.07 \pm 0.18$ in the low-field and low-temperature limit.

The most reliable measurement of the absolute value of the penetration depth is likely to be from neutron diffraction and in 2003 Cubitt et al. [3] found that at $2 \mathrm{~K}$, the extrapolated low-field $(<100 \mathrm{mT})$ value was $\Lambda_{a b}=82 \pm 2 \mathrm{~nm}$, which is close to our value. As samples grow as thin plates in the $a b$ plane, Cubitt et al. did not have direct access to $\Lambda_{c}$ and so acquired diffraction patterns with vortices tilted at $45^{\circ}$ with respect to the $c$ axis. It was uncertain whether the formula used to calculate the anisotropy was valid for a two-band superconductor [3] but data acquired at $2 \mathrm{~K}$ between $0.2-0.5 \mathrm{~T}$ indicated that $\gamma_{\Lambda}$ varied with field and its extrapolated value at low field was $\gamma_{\Lambda}=1.1 \pm 0.3$. In 2006, Pal et al. [25] used a different neutron diffraction technique to give $\gamma_{\Lambda}=1.1 \pm 0.1$ at $4.9 \mathrm{~K}$. Combining this with the neutron value for $\Lambda_{a b}$ gives $\Lambda_{c}=90 \pm 8 \mathrm{~nm}$, which agrees with our value of $\Lambda_{c}=$ $102 \pm 15 \mathrm{~nm}$. The anisotropy we obtain is close to the value of 1.01 calculated from first principles by Golubov et al. [26] in the clean limit, but in common with Fletcher et al. [24], we find penetration depths approximately twice as large as predicted.

Cubitt et al. interpreted their data assuming that the coherence length did not vary with field. If it did, the value they obtained, $\xi_{a b}=8 \pm 1 \mathrm{~nm}$, would apply only at high field $(>0.8 \mathrm{~T})$. This is close to the value of $10 \mathrm{~nm}$ found from the upper critical field [27].

Eskildsen et al. [28] measured the coherence length in 2002 using scanning tunneling microscopy (STM) to measure the width of vortex cores, scanning the $a b$ plane with tunneling in $c$. As the $\sigma$ carriers are confined to the $a b$ planes, the tunnelling current came almost exclusively from the $\pi$ band giving $\xi_{\pi}=38.8 \pm 0.7 \mathrm{~nm}$ at $0.32 \mathrm{~K}$ in a field of $50 \mathrm{mT}$ (after adjusting for their slightly different model for the core). This agrees with our value of $\xi_{c}=35 \pm 10 \mathrm{~nm}$ at $10.8 \mathrm{~K}$ and $4.8 \mathrm{mT}$ and supports this larger value at low field.

\section{SUMMARY AND CONCLUSIONS}

We have described a new method to measure the penetration depth and coherence length of a superconductor in all directions at low applied magnetic field using transmission electron microscopy. The measurement does not need large-scale facilities and required one day to thin and mount the sample and another day to take the images required. The experiment was performed on a very small sample: $30 \times 15 \mu \mathrm{m}^{2}$ and $200-\mathrm{nm}$ thick so this method could prove useful for superconductors where only very small single crystals are available, as is the case for some iron-based superconductors. It is also useful if the penetration depth and coherence length vary with field, as is the case for $\mathrm{MgB}_{2}$, as the measurement is made at very low fields, which can be difficult to access using other techniques.

For a sample of $\mathrm{MgB}_{2}$ cut in the $a c$ plane and tilted to $45 \pm 5^{\circ}$ about the $a$ axis, we obtained $\Lambda_{a b}=100 \pm 35 \mathrm{~nm}$ and $\Lambda_{c}=120 \pm 15 \mathrm{~nm}$ at $10.8 \mathrm{~K}$ in a field of $4.8 \mathrm{mT}$. The large error in $\Lambda_{a b}$ is a consequence of tilting the sample about the crystallographic $a$ axis. Had it been tilted about $c$ instead, the errors on $\Lambda_{a b}$ and $\Lambda_{c}$ would be reversed. We obtained a more precise value of $\Lambda_{a b}=107 \pm 8 \mathrm{~nm}$ at $10.8 \mathrm{~K}$ in our previous paper [4] in which the sample was cut in the $a b$ plane. Using this value gives $\Lambda_{a b}=107 \pm 8 \mathrm{~nm}, \Lambda_{c}=120 \pm 15 \mathrm{~nm}$, $\xi_{a b}=39 \pm 11 \mathrm{~nm}$, and $\xi_{c}=35 \pm 10 \mathrm{~nm}$, which agree well with measurements made using other techniques discussed in Sec. V.

Obtaining the most precise values for the penetration depths and coherence lengths in all directions using this technique requires taking images of the vortex lattice with the sample tilted in more than one direction. It might be thought that the sample could be mounted in the plane of the support grid and tilted with the microscope goniometer, first about $a$ and then about $c$. However, the design of conventional liquid helium cooled holders does not allow tilting to an angle higher than $\alpha=25^{\circ}$, so the sample was instead mounted to the support grid at $45 \pm 5^{\circ}$ to give sufficient contrast in the image. Thus, to investigate a new superconductor and obtain the most accurate measurement of the penetration depth and coherence length in all directions, it would be best to cut two samples and mount both to the grid, one tilted about $a$ and the other tilted about $c$.

We used the Ginzburg-Landau model for the magnetic structure of flux vortices but as $\mathrm{MgB}_{2}$ is a two-band superconductor, the vortices may well have a more complex structure as described in Ref. [29]. We obtained good fits to the vortex images and there was no indication of a more complex structure within the accuracy of the technique but our simulation scheme would allow any model for the vortex structure to be used for image simulations provided that the magnetic vector potential for a vortex in an infinitely thick superconductor is known.

\section{ACKNOWLEDGMENTS}

This work was funded by the Royal Society. Work at Eidgenössische Technische Hochschule, Zürich was supported by the Swiss National Science Foundation and the National Center of Competence in Research programme "Materials with Novel Electronic Properties." 
[1] J. Nagamatsu, N. Nakagawa, T. Kuranaka, Y. Zenitani, and J. Akimittsu, Superconductivity at $39 \mathrm{~K}$ in magnesium diboride, Nature (London) 410, 63 (2001).

[2] H. J. Choi, D. Roundy, H. Sun, M. L. Cohen, and S. G. Louie, The origin of the anomalous superconducting properties of $\mathrm{MgB}_{2}$, Nature (London) 418, 758 (2002).

[3] R. Cubitt, M. R. Eskildsen, C. D. Dewhurst, J. Jun, S. M. Kazakov, and J. Karpinski, Effects of two-band superconductivity on the flux-line lattice in magnesium diboride, Phys. Rev. Lett. 91, 047002 (2003).

[4] J. C. Loudon, C. J. Bowell, N. D. Zhigadlo, J. Karpinski, and P. A. Midgley, Magnetic structure of individual flux vortices in superconducting $\mathrm{MgB}_{2}$ derived using transmission electron microscopy, Phys. Rev. B 87, 144515 (2013).

[5] K. Harada, T. Matsuda, J. Bonevich, M. Igarashi, S. Kondo, G. Pozzi, U. Kawabe, and A. Tonomura, Real-time observation of vortex lattices in a superconductor by electron microscopy, Nature (London) 360, 51 (1992).

[6] http://youtu.be/0v-aBx0-kbw.

[7] M. Beleggia, G. Pozzi, J. Masuko, N. Osakabe, K. Harada, T. Yoshida, O. Kamimura, H. Kasai, T. Matsuda, and A. Tonomura, Interpretation of Lorentz microscopy observations of vortices in high-temperature superconductors with columnar defects, Phys. Rev. B 66, 174518 (2002).

[8] J. R. Clem, Vortices in superconducting films, AIP Conf. Proc. 58, 245 (1980).

[9] R. A. Klemm and J. R. Clem, Lower critical field of an anisotropic type-II superconductor, Phys. Rev. B 21, 1868 (1980).

[10] M. Beleggia and G. Pozzi, Observation of superconducting fluxons by transmission electron microscopy: A Fourier space approach to calculate the electron optical phase shifts and images, Phys. Rev. B 63, 054507 (2001).

[11] J. R. Clem, Z. Hao, L. Dobrosavlijević, and Z. Radović, Vortices in anisotropic type-II superconductors, J. Low Temp. Phys. 88, 213 (1992).

[12] J. F. Annett, Superconductivity, Superfluids and Condensates (Oxford University Press, Oxford, 2004).

[13] V. L. Ginzburg and L. D. Landau, On the theory of superconductivity, Zh. Eksp. Teor. Fiz. 20, 1064 (1950) [English translation in Collected Papers of L. D. Landau, edited by D. ter Haar (Pergamon Press, Oxford, 1965), Chap. 73].

[14] J. R. Clem, Simple model for the vortex core in a type II superconductor, J. Low Temp. Phys. 18, 427 (1975).

[15] E. H. Brandt, Ginzburg-Landau vortex lattice in superconductor films of finite thickness, Phys. Rev. B 71, 014521 (2005).
[16] J. Karpinski, S. M. Kazakov, J. Jun, M. Angst, R. Puzniak, A. Wisniewski, and P. Bordet, Single crystal growth of $\mathrm{MgB}_{2}$ and thermodynamics of $\mathrm{Mg}-\mathrm{B}-\mathrm{N}$ system at high pressure, Physica C 385, 42 (2003).

[17] J. Karpinski, N. D. Zhigadlo, S. Katrych, R. Puzniak, K. Rogacki, and R. Gonnelli, Single crystals of $\mathrm{MgB}_{2}$ : Synthesis, substitutions and properties, Physica C 456, 3 (2007).

[18] N. D. Zhigadlo, S. Katrych, J. Karpinski, B. Batlogg, F. Bernardini, S. Massidda, and R. Puzniak, Influence of $\mathrm{Mg}$ deficiency on crystal structure and superconducting properties in $\mathrm{MgB}_{2}$ single crystals, Phys. Rev. B 81, 054520 (2010).

[19] D. B. Williams and C. B. Carter, Transmission Electron Microscopy (Springer, New York, 1996), Chap. 28.

[20] R. F. Egerton, Electron energy-loss spectroscopy in the TEM, Rep. Prog. Phys. 72, 016502 (2009).

[21] D. Rez, P. Rez, and I. Grant, Dirac-Fock calculations of X-ray scattering factors and contributions to the mean inner potential for electron scattering, Acta. Cryst. A50, 481 (1994).

[22] W. H. Press, B. P. Flannery, S. A. Teukolsky, and T. T. Vetterling, Numerical Recipes (Cambridge University Press, Cambridge, 1992), Chap. 10.4.

[23] J. C. Loudon, C. J. Bowell, N. D. Zhigadlo, J. Karpinski, and P. A. Midgley, Imaging flux vortices in $\mathrm{MgB}_{2}$ using transmission electron microscopy, Physica C 474, 18 (2012).

[24] J. D. Fletcher, A. Carrington, O. J. Taylor, S. M. Kazakov, and J. Karpinski, Temperature-dependent anisotropy of the penetration depth and coherence length of $\mathrm{MgB}_{2}$, Phys. Rev. Lett. 95, 097005 (2005).

[25] D. Pal, L. DeBeer-Schmitt, T. Bera, R. Cubitt, C. D. Dewhurst, J. Jun, N. D. Zhigadlo, J. Karpinski, V. G. Kogan, and M. R. Eskildsen, Measuring the penetration depth anisotropy in $\mathrm{MgB}_{2}$ using small-angle neutron scattering, Phys. Rev. B 73, 012513 (2006).

[26] A. A. Golubov, A. Brinkman, O. V. Dolgov, J. Kortus, and O. Jepsen, Multiband model for penetration depth in $\mathrm{MgB}_{2}$, Phys. Rev. B 66, 054524 (2002).

[27] M. R. Eskildsen, M. Kugler, G. Levy, S. Tanaka, J. Jun, S. M. Kazakov, J. Karpinski, and Ø. Fischer, Scanning tunneling spectroscopy on single crystal $\mathrm{MgB}_{2}$, Physica C 385, 169 (2003).

[28] M. R. Eskildsen, M. Kugler, S. Tanaka, J. Jun, S. M. Kazakov, J. Karpinski, and Ø. Fischer, Vortex imaging in the $\pi$ band of magnesium diboride, Phys. Rev. Lett. 89, 187003 (2002).

[29] E. Babaev and J. Carlström, Type-1.5 superconductivity in twoband systems, Physica C 470, 717 (2010). 